\title{
openheart Relationship between procedural characteristics and cerebrovascular events after transcatheter aortic valve replacement
}

\author{
Kashish Goel, ${ }^{1}$ Vuyisile T Nkomo, ${ }^{1}$ Joshua P Slusser, ${ }^{2}$ Ryan Lennon, ${ }^{2}$ \\ Robert D Brown, ${ }^{3}$ Kevin L Greason, ${ }^{4}$ David R Holmes Jr ${ }^{1}$
}

\begin{abstract}
- Additional material is published online only. To view, please visit the journal online (http://dx.doi.org/10.1136/ openhrt-2018-000816).
\end{abstract}

To cite: Goel K, Nkomo VT, Slusser JP, et al. Relationship between procedural characteristics and cerebrovascular events after transcatheter aortic valve replacement. Open Heart 2018;5:e000816. doi:10.1136/ openhrt-2018-000816

Received 6 March 2018 Revised 19 May 2018 Accepted 13 June 2018

\section{Check for updates}

C) Author(s) (or their employer(s)) 2018. Re-use permitted under CC BY-NC. No commercial re-use. See rights and permissions. Published by BMJ.

'Department of Cardiovascular Diseases, Mayo Clinic, Rochester, Minnesota, USA ${ }^{2}$ Division of Biomedical Statistics and Informatics, Mayo Clinic, Rochester, Minnesota, USA ${ }^{3}$ Department of Neurology, Mayo Clinic, Rochester, Minnesota, USA

${ }^{4}$ Department of Cardiovascular Surgery, Mayo Clinic, Rochester Minnesota, USA

Correspondence to

David R Holmes Jr; holmes. david@mayo.edu

\section{ABSTRACT}

Objectives The objective was to assess the impact of procedural characteristics on risk of stroke or transient ischaemic attack (TIA) after transcatheter aortic valve replacement (TAVR)

Methods We included 370 consecutive patients who underwent balloon-expandable TAVR from 1 November 2008 to 30 June 2014. Procedural characteristics that may be associated with stroke/TIA were assessed. The primary outcome was stroke/TIA at 30 days. A propensity score was constructed using a logistic regression model with 29 parameters. Cox proportional hazards models were used with a propensity score covariate.

Results Mean age was $80.9 \pm 7.9$ years and mean Society of Thoracic Surgeons score was $8.3 \pm 5.0$. The total number of balloon dilations ranged from 2 to 7 . Out of 370 patients, 13 patients (3.5\%) suffered stroke/TIA in the first 30 days after TAVR. In univariate analysis, postdeployment balloon dilation (PD) (HR 3.8, 95\% Cl 1.24 to 11.61 ; $\mathrm{p}=0.02$ ) and emergent cardiopulmonary bypass (CPB) (HR 9.66, $95 \% \mathrm{Cl} 2.66$ to $35.15 ; \mathrm{p}<0.001$ ) were significantly associated with 30 -day stroke/TIA. In the multivariable Cox-proportional hazards model, PD (HR 4.95, 95\% Cl 1.02 to $24.03 ; p=0.04$ ) and emergent CPB (HR 7.15, 95\% $\mathrm{Cl} 1.39$ to $36.89 ; \mathrm{p}=0.02$ ) were independently associated with increased risk of 30-day stroke/TIA after adjusting for propensity score, total number of balloon dilations and periprosthetic regurgitation.

Conclusion Postdilation as compared with total number of dilations, and emergent CPB were independently associated with increased risk of clinical neurological events in the first 30 days after TAVR. Reduction in balloon postdilation with appropriate valve sizing may reduce the risk of stroke or TIA after TAVR.

\section{INTRODUCTION}

Transcatheter aortic valve replacement (TAVR) is approved for high-risk and inoperable patients with severe aortic stenosis and has been performed in $>30000$ patients in the USA. ${ }^{1}$ Recent trials have reported the safety and efficacy of TAVR as in intermediate risk patients. ${ }^{2}$ Although procedural success rates have been very high, one of the major

\section{Key questions}

What is already known about this subject?

- Postdilation is associated with increased risk of clinical stroke in patients undergoing transcatheter aortic valve replacement (TAVR).

What does this study add?

- This study shows that postdilation as compared with total number of dilations during TAVR is associated with increased risk of clinical neurological events.

How might this impact on clinical practice?

- This study identifies postdilation and use of emergent cardiopulmonary bypass as independent predictors of clinical neurological events in patients undergoing balloon-expandable TAVR.

- Appropriate valve sizing and foregoing postdilation may be important to reduce clinical neurological events after TAVR

concerns is the occurrence of stroke after TAVR, ${ }^{3-5}$ especially as this technology is likely to be applied in intermediate and potentially low-risk patients in the future.

National data from the American College of Cardiology transcatheter valve therapy registry showed that the incidence of stroke after TAVR was $2.5 \%$ at 30 days. ${ }^{1}$ MRI-based studies report that almost two-thirds of the patients undergoing TAVR develop ischaemic cerebral lesions within 1 week of the procedure ${ }^{67}$ Balloon dilatation prior to,${ }^{89}$ during and after valve deployment ${ }^{10-12}$ could be one of the explanations for high incidence of stroke or transient ischaemic attack (TIA) (stroke/TIA) in patients undergoing TAVR, presumably due to embolisation of calcific material from the aortic valve. However, there is limited data regarding this possible association and controversies still remain regarding its safety. ${ }^{13}$ In addition, it is unknown if the number of total dilations (prevalve, during and postvalve insertion), use and urgency of cardiopulmonary bypass (CPB) 
and procedural duration increase the risk of cerebrovascular events after TAVR.

The objective of this study was to assess the impact of various procedural characteristics on the risk of 30-day stroke/TIA in patients undergoing balloon-expandable TAVR.

\section{METHODS}

\section{Study population}

The Mayo Clinic cardiovascular surgery database contains prospectively collected data on all patients who underwent TAVR at Mayo Clinic, Rochester, Minnesota, USA. For the present study, 370 consecutive patients who underwent TAVR from 1 November 2008 to 30 June 2014 were included. Patients who died in the operating room $(n=5)$, converted to open aortic valve replacement surgery $(n=6)$, had valve-in-valve procedure for previous bioprosthetic aortic valve degeneration $(n=11)$ and those who did not give authorisation to use their medical records for research purposes $(n=4)$ were excluded. All patients gave informed consent for the procedure. The patient selection for TAVR was based on the recommendations of a multidisciplinary heart team including cardiac surgeons, interventional cardiologists and valve specialists.

The operative reports were reviewed to record the procedural characteristics including number of balloon valvuloplasties prior to valve deployment (prevalve), number of dilations during valve deployment (two if a second valve was used due to any reason), postdeployment balloon dilations (PD), total number of right ventricular (RV) pacing runs, procedural time, type of access and use and urgency of CPB. The mean arterial pressure was assessed at the start of the procedure. Demographic, clinical, angiographic, procedural and medication data available in the data registry were assessed for all patients. Data were recorded in keeping with definitions set forth in The Society of Thoracic Surgeons (STS) Adult Cardiac Surgery Database, V.2.73. ${ }^{14}$ Pre-TAVR transthoracic echocardiograms were assessed for all patients. Intraoperative transoesophageal echocardiogram (TEE) was used to assess the degree of paravalvular regurgitation (PVR) after TAVR. All the TEEs were performed and read by level III trained and experienced interventional echocardiography physicians. Pre-TAVR CT scan reports were used to obtain the CT aortic valve calcium score by the method by Agatston et $^{1 l^{15}}$ and the degree of aortic atherosclerosis was ascertained by the reading cardiovascular radiologist.

\section{Outcomes}

The primary outcome of the study was 30-day incidence of stroke/TIA. Secondary outcomes were 30-day and long-term all-cause mortality.

\section{Definitions}

Stroke/TIA was defined retrospectively according to the Valve Academic Research Consortium-2 Consensus statement. ${ }^{16}$ This included any acute episode of a focal or global neurological deficit with at least one of the following: change in the level of consciousness, hemiplegia, hemiparesis, numbness or sensory loss affecting one side of the body, dysphasia or aphasia, hemianopia, amaurosis fugax or other neurological signs or symptoms consistent with stroke. TIA was defined when the duration of symptoms lasted $<24$ hours and neuroimaging was not consistent with an infarct. All patients were evaluated by a consultant neurologist at the time of the event and diagnosis was confirmed by clinical assessment. Additionally, all patients had either had a head CT, MRI or both performed as part of their evaluation. All of the strokes were ischaemic in aetiology, including one watershed infarct. Patients who did not have a clinical cerebrovascular event were not evaluated by a neurologist.

\section{Statistical analysis}

Continuous variables are summarised as mean (SD); discrete variables are presented as frequency (percentage). Cox proportional hazards models were used to estimate the HRs for association with 30-day and long-term outcomes. The following were explored in unadjusted models as potential predictors for stroke/TIA and death: total number of balloon dilations during TAVR, PD, procedural time, use of CPB, mean arterial pressure, severity of atherosclerosis, aortic valve calcium score on CT, sex and history of stroke/TIA. After PD was identified as having a significant association with 30-day stroke/TIA, a propensity score for any PD after TAVR was constructed using a logistic regression model with 29 parameters (see online supplemental files). A matching analysis based on the propensity score was attempted but too few events (one 30-day stroke/ TIA) remained in the set of matched cases and controls for inference. A Cox proportional hazards model with PD and the propensity score was fit to estimate an adjusted association between PD and 30-day stroke/TIA. Additionally, the number of valve inflations, periprosthetic aortic regurgitation and emergent $\mathrm{CPB}$ were added as covariates to test the robustness of the association. The Firth modification for maximum likelihood estimation was used to handle convergence issues. Cumulative incidence estimators in the presence of competing risks were used to estimate the risk of stroke/TIA on follow-up. Kaplan-Meier methods were used for survival. Fine and Grey models for the outcome of stroke/TIA were also attempted to account for the competing risk of death, but gave nearly identical HRs as the Cox model and are not presented. All tests were two-sided and a $p$ value $<0.05$ was considered to be statistically significant. SAS V.9 (SAS Institute, Cary, North Carolina, USA) was used for all analyses.

\section{RESULTS \\ Baseline, clinical and procedural characteristics}

Table 1 details the descriptive characteristics of the study population $(n=370)$. Mean age of the study population was $80.9 \pm 7.9$ years, mean STS score was $8.3 \pm 5.0$, mean aortic valve gradient was $49.4 \pm 13.2 \mathrm{~mm} \mathrm{Hg}$ and mean aortic valve area was $0.8 \pm 0.2 \mathrm{~cm}^{2}$. 
Table 1 Descriptive characteristics of the study population

\begin{tabular}{|c|c|}
\hline Variables & $\mathrm{n}=\mathbf{3 7 0}$ \\
\hline Age (years) & $80.9(7.9)$ \\
\hline Males, n (\%) & $207(56 \%)$ \\
\hline Prior atrial fibrillation, $\mathrm{n}(\%)$ & $154(42 \%)$ \\
\hline STS score & $8.3(5.0)$ \\
\hline NYHA class IV, n (\%) & $104(28 \%)$ \\
\hline Diabetes, $n(\%)$ & $153(41 \%)$ \\
\hline Dyslipidemia, n (\%) & $333(90 \%)$ \\
\hline Hypertension, $\mathrm{n}(\%)$ & $333(90 \%)$ \\
\hline History of stroke/TIA, n (\%) & $33(9 \%)$ \\
\hline Peripheral vascular disease, $\mathrm{n}(\%)$ & $220(59 \%)$ \\
\hline Moderate/severe renal disease, $\mathrm{n}(\%)$ & $246(66 \%)$ \\
\hline Prior CABG, $n(\%)$ & $161(44 \%)$ \\
\hline Previous MI, $\mathrm{n}(\%)$ & $137(37 \%)$ \\
\hline Prior valve intervention, $\mathrm{n}(\%)$ & $73(20 \%)$ \\
\hline \multicolumn{2}{|l|}{ Pre-TAVR medications } \\
\hline Antiarrhythmics, n (\%) & $32(9 \%)$ \\
\hline Aspirin, $n(\%)$ & $271(73 \%)$ \\
\hline Coumadin, n (\%) & $22(6 \%)$ \\
\hline \multicolumn{2}{|l|}{ Echocardiographic parameters } \\
\hline Ejection fraction & $56.3(13.5)$ \\
\hline Pulmonary artery systolic pressure $(\mathrm{mm} \mathrm{Hg})$ & $43.9(14.3)$ \\
\hline Left atrial volume index $\left(\mathrm{cc} / \mathrm{m}^{2}\right)$ & $48.9(14.9)$ \\
\hline Aortic valve area & $0.8(0.2)$ \\
\hline Mean systolic aortic valve gradient & $49.4(13.2)$ \\
\hline Moderate/severe MR (\%) & $96(27 \%)$ \\
\hline \multicolumn{2}{|l|}{ CT scan } \\
\hline \multicolumn{2}{|l|}{ Severity of aortic atherosclerosis, $n(\%)$} \\
\hline None, trivial or mild & $164(53 \%)$ \\
\hline Mild-to-moderate or moderate & $106(34 \%)$ \\
\hline Moderate-to-severe or severe & $40(13 \%)$ \\
\hline CT aortic valve calcium score & $2966.5(1633.4)$ \\
\hline
\end{tabular}

CABG, coronary artery bypass surgery; MR, mitral regurgitation; MI, myocardial infarction; NYHA, New York Heart Association; STS, Society of Thoracic Surgeons; TIA, transient ischaemic attack; TAVR, transcatheter aortic valve replacement.

Table 2 summarises the procedural characteristics of the patients. The total number of dilations including prevalve, valve and postvalve deployment ranged from 2 to 7 , with $6 \%(\mathrm{n}=23)$ being treated with $\geq 4$ total dilations. All patients except one underwent pre-TAVR balloon aortic valvuloplasty. A total of 16 patients (4\%) underwent placement of two or more valves during the same procedure. Second valves were deployed due to persistent moderate-to-severe or severe periprosthetic regurgitation $(n=11)$, severe prosthetic regurgitation $(n=3)$, device migration into the ascending aorta $(\mathrm{n}=1)$ and prosthetic valve malfunction $(\mathrm{n}=1)$. The overall incidence of PD was $14 \%(\mathrm{n}=53)$. CPB was used in 16 patients (4\%) and intra-aortic balloon pump
Table 2 Procedural characteristics of the study population

\begin{tabular}{|c|c|}
\hline Procedural characteristics & $\mathrm{n}=\mathbf{3 7 0}$ \\
\hline \multicolumn{2}{|l|}{ Number of pre(TAVR BAV, $\mathrm{n}(\%))$} \\
\hline 1 & $341(91 \%)$ \\
\hline 2 & $23(6 \%)$ \\
\hline 3 & $5(1 \%)$ \\
\hline 4 & $1(0 \%)$ \\
\hline \multicolumn{2}{|l|}{ Number of valves, $\mathrm{n}(\%)$} \\
\hline 1 & $354(97 \%)$ \\
\hline 2 & $15(4 \%)$ \\
\hline 3 & $1(0 \%)$ \\
\hline \multicolumn{2}{|c|}{ Number of post(dilations after TAVR, $\mathrm{n}(\%))$} \\
\hline 0 & $317(86 \%)$ \\
\hline 1 & $49(13 \%)$ \\
\hline 2 & $3(1 \%)$ \\
\hline 3 & $1(1 \%)$ \\
\hline \multicolumn{2}{|c|}{ Total number of dilations during TAVR, $n(\%)$} \\
\hline $1-2$ & $293(79 \%)$ \\
\hline 3 & $56(16 \%)$ \\
\hline $4+$ & $23(6 \%)$ \\
\hline Procedural time (min) & $111.5(43.3)$ \\
\hline \multicolumn{2}{|c|}{ Periprosthetic aortic regurgitation, $\mathrm{n}(\%)$} \\
\hline None & $44(12 \%)$ \\
\hline Trivial & $183(49 \%)$ \\
\hline Mild & $99(27 \%)$ \\
\hline Mild/moderate & $31(8 \%)$ \\
\hline Moderate & $13(4 \%)$ \\
\hline \multicolumn{2}{|l|}{ Cardiopulmonary bypass, $\mathrm{n}(\%)$} \\
\hline None & $354(96 \%)$ \\
\hline Prophylactic & $4(1 \%)$ \\
\hline Emergent & $12(3 \%)$ \\
\hline Intra(aortic balloon pump, $\mathrm{n}(\%)$ ) & $6(2 \%)$ \\
\hline Systolic blood pressure (mm Hg) & $126.6(26.3)$ \\
\hline Diastolic blood pressure $(\mathrm{mm} \mathrm{Hg})$ & $61.2(14)$ \\
\hline Mean arterial pressure $(\mathrm{mm} \mathrm{Hg})$ & $83(15.9)$ \\
\hline Intraoperative blood products (\%) & $126(34 \%)$ \\
\hline \multicolumn{2}{|l|}{ Valve size $(\mathrm{mm}), \mathrm{n}(\%)$} \\
\hline 23 & $127(34 \%)$ \\
\hline 26 & $213(57 \%)$ \\
\hline 29 & $30(8 \%)$ \\
\hline \multicolumn{2}{|l|}{ Access, $n(\%)$} \\
\hline Transapical & $168(45 \%)$ \\
\hline Transfemoral & $182(49 \%)$ \\
\hline Other & $20(5 \%)$ \\
\hline
\end{tabular}

BAV, balloon aortic valvuloplasty; TAVR, transcatheter aortic valve replacement.

was used in 6 patients (2\%). Prophylactic CPB was used in $4(1 \%)$ patients and emergent $\mathrm{CPB}$ was required in 12 (3\%) patients. The reasons for emergent CPB were severe PVR $(n=4)$, haemodynamic collapse after transapical access $(\mathrm{n}=4)$, balloon valvuloplasty prior to valve deployment $(\mathrm{n}=1)$ and due to other causes $(\mathrm{n}=3)$. 
Table 3 Univariate analysis of procedural characteristics and 30-day outcomes

\begin{tabular}{|c|c|c|c|c|}
\hline \multirow[b]{2}{*}{ Variables } & \multicolumn{2}{|c|}{ 30-Day stroke/TIA } & \multicolumn{2}{|l|}{ 30-Day mortality } \\
\hline & HR (95\% Cl) & P values & HR (95\% Cl) & P values \\
\hline Total number of dilations during TAVR & 1.58 (0.95 to 2.61$)$ & 0.076 & 1.20 (0.60 to 2.38$)$ & 0.61 \\
\hline Postdilations after TAVR & 3.80 (1.24 to 1.61$)$ & 0.019 & $1.08(0.24$ to 4.85$)$ & 0.93 \\
\hline Emergent cardiopulmonary bypass & 9.66 (2.66 to 35.15$)$ & $<0.001$ & $2.41(0.31$ to 18.56$)$ & 0.4 \\
\hline Severity of atherosclerosis & $1.88(0.95$ to 3.75$)$ & 0.072 & 1.26 (0.60 to 2.64$)$ & 0.54 \\
\hline CT aortic valve calcium score (per 1000) & 0.96 (0.66 to 1.41$)$ & 0.853 & $1.04(0.75$ to 1.44$)$ & 0.82 \\
\hline Procedural time (per $30 \mathrm{~min})$ & $1.29(0.96$ to 1.74$)$ & 0.093 & 1.50 (1.17 to 1.92$)$ & 0.001 \\
\hline Transfemoral vs transapical access & $0.77(0.23$ to 2.51$)$ & 0.658 & $0.76(0.23$ to 2.48$)$ & 0.65 \\
\hline Mean blood pressure (per $10 \mathrm{~mm} \mathrm{Hg}$ ) & 1.05 (0.75 to 1.48$)$ & 0.756 & 0.98 (0.69 to 1.38$)$ & 0.89 \\
\hline Female sex & 1.09 (0.37 to 3.25$)$ & 0.876 & $0.57(0.18$ to 1.85$)$ & 0.35 \\
\hline History of stroke/TIA & 0.37 (0.02 to 6.97$)$ & 0.508 & $0.38(0.02$ to 7.06$)$ & 0.51 \\
\hline
\end{tabular}

TAVR, transcatheter aortic valve replacement; TIA, transient ischaemic attack.

\section{Procedural characteristics and clinical cerebrovascular events}

Of the 370 patients undergoing TAVR, $13(3.5 \%)$ patients suffered stroke/TIA during the first 30 days after the procedure, $8(2.1 \%)$ of which were strokes. Results of the univariate analysis for the procedural characteristics associated with stroke/TIA are presented in table 3. PD (HR $3.8,95 \%$ CI 1.24 to $11.61 ; \mathrm{p}=0.02)$ and emergent $\mathrm{CPB}$ (HR 9.66, $95 \%$ CI 2.66 to 35.15 ; $\mathrm{p}<0.001$ ) were associated with a significantly increased risk of stroke/TIA in the first 30 days. There was a non-significant trend towards increased 30-day stroke/TIA with the total number of dilations (HR $1.58,95 \%$ CI 0.95 to $2.61 ; \mathrm{p}=0.08$ ), longer procedural time (HR per $30 \mathrm{~min}, 1.29$, 95\% CI 0.96 to $1.74 ; \mathrm{p}=0.09$ ) and severity of atherosclerosis (moderate-to-severe-HR 1.88 ; $95 \%$ CI 0.95 to 3.75 ; $\mathrm{p}=0.07$ ).

Figure 1A shows the Kaplan-Meier curves for incidence of 30-day stroke/TIA stratified by PD. The incidence of stroke/TIA in the first 30 days after TAVR was higher in patients who underwent PD compared with those without PD (9.4\% vs $2.2 \% ; \mathrm{p}=0.02)$. The incidence of stroke alone was also higher in PD $(5.6 \% ; \mathrm{n}=3 / 53)$ versus non-PD $(1.6 \% ; n=5 / 317)$ patients. After adjusting for propensity score only, PD remained significantly associated with the 30-day stroke/TIA (HR 5.21, 95\% CI 1.16 to 23.34; $\mathrm{p}=0.03)$. In the final multivariate model, PD (HR 4.95, 95\% CI 1.02 to 24.03; $\mathrm{p}=0.04$ ) was independently associated with 30-day risk of stroke/TIA after adjusting for the propensity score, total number of balloon dilations, periprosthetic regurgitation and emergent CPB (table 4).

Figure 1B shows the Kaplan-Meier curves for 30-day stroke/TIA stratified by use and urgency of CPB. In the final multivariable model, emergent CPB during TAVR was associated with a significantly higher incidence of stroke/TIA (HR 7.15, 95\% CI 1.39 to 36.89; p=0.02) (table 4).
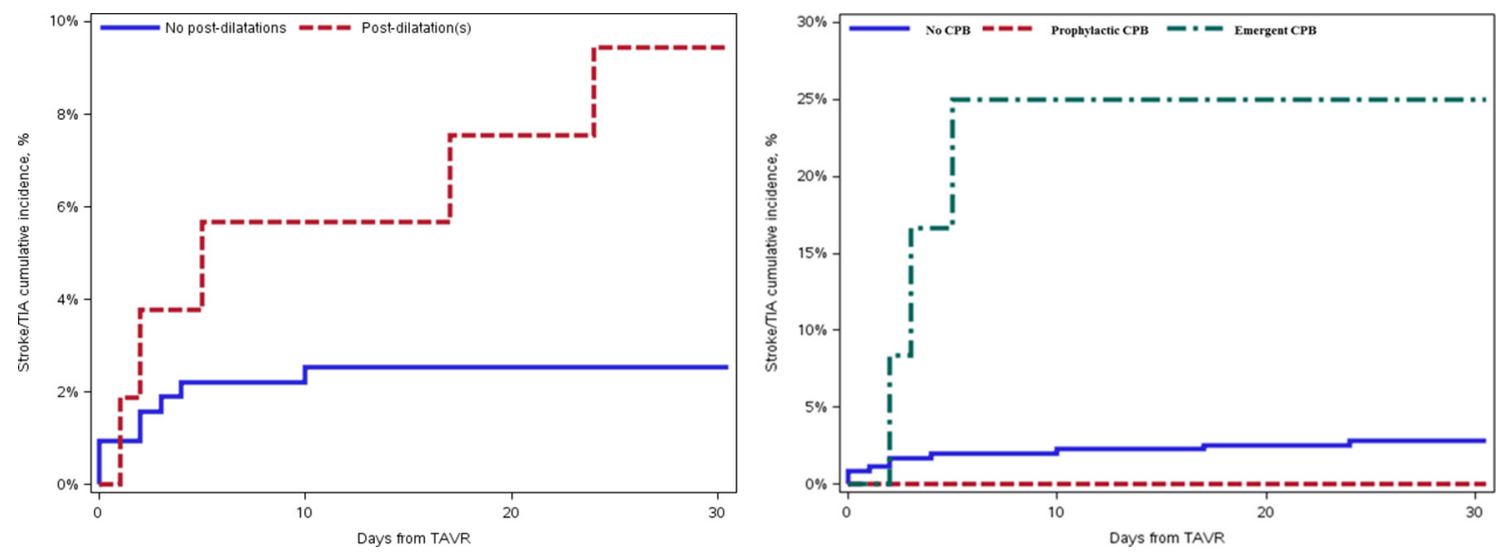

Figure 1 (A) Kaplan-Meier curves for 30-day stroke/TIA stratified by postdeployment balloon dilation. Red line represents postdeployment balloon dilation and blue line represents those who did not undergo postdeployment balloon dilation during TAVR. Log-rank test $p$ value=0.019. (B) Kaplan-Meier curves for 30-day stroke/TIA stratified by the use and urgency of cardiopulmonary bypass. Green line represents emergent cardiopulmonary bypass, red line represents prophylactic CPB and blue line represents those who did not require CBP during TAVR. Log-rank test $p<0.001$. CPB, cardiopulmonary bypass; TAVR, transcatheter aortic valve replacement; TIA, transient ischaemic attack. 
Table 4 Propensity-adjusted final multivariable Cox model for 30-day stroke/TIA

\begin{tabular}{lcl}
\hline Variables & HR (95\% Cl) & P \\
\hline Postdilations after TAVR & $4.95(1.02$ to 24.03$)$ & 0.04 \\
\hline Emergent cardiopulmonary bypass & $7.15(1.39$ to 36.89$)$ & 0.02 \\
\hline Propensity score for PD & $0.79(0.55$ to 1.13$)$ & 0.19 \\
\hline Periprosthetic AR & $1.42(0.73$ to 2.79$)$ & 0.3 \\
\hline Total number of balloon dilations & $1.27(0.13$ to 12.45$)$ & 0.83 \\
\hline
\end{tabular}

$\mathrm{AR}$, aortic regurgitation; $\mathrm{PD}$, postdeployment balloon dilation; TAVR, transcatheter aortic valve replacement.

\section{Procedural characteristics and all-cause mortality}

Of the procedural characteristics assessed, only procedural time was associated with an increased risk of 30-day mortality (HR $1.5,95 \%$ CI 1.2 to $1.9 ; \mathrm{p}=0.001$ ). Postdilation (HR 1.08, 95\% CI 0.24 to 4.85; p=0.93) or CPB (HR $1.79,95 \%$ CI 0.23 to $13.76 ; \mathrm{p}=0.58)$ did not increase the risk of all-cause mortality at 30 days. In propensity-score adjusted analysis, PD (HR 1.003, 95\% CI 0.53 to 1.91 ; $\mathrm{p}=0.93$ ) was not associated with long-term mortality (see online supplemental figure). In-hospital outcomes are reported in the online supplemental table 3 .

\section{DISCUSSION}

Postdilation was independently associated with increased risk of stroke/TIA at 30 days after TAVR, as compared with total number of balloon dilations or RV pacing runs. In addition, emergent CPB were independently associated with an increased risk of stroke/TIA at 30 days. The total number of balloon dilations (total dilations including prevalve, valve inflations and PD), severity of aortic atherosclerosis on CT scan and procedural time showed a trend towards increased risk of 30-day stroke/ TIA in univariate analysis only. Lastly, PD was not associated with short-term or long-term mortality after TAVR.

TAVR is approved for high-risk and inoperable patients and $>150000$ procedures have been performed worldwide. ${ }^{17}$ The risk of mortality is similar to surgical AVR; however, the rate of cerebrovascular events is higher. ${ }^{18} 19$ Often, patients undergoing TAVR have inherent risk factors for stroke such as advanced age, hypertension, hyperlipidaemia and carotid and aortic atherosclerosis, which place them at a higher risk of cerebrovascular events. Preventable causes in patients undergoing TAVR include procedural factors associated with stroke/TIA. Previous studies have focused primarily on clinical risk factors or only a few procedural characteristics such as PD, type of access or valve type ${ }^{11} 17$; however, detailed evaluation of other procedural factors and their association with postprocedure cerebrovascular events has not been performed. We systematically analysed our single-centre data to assess the relative risk of stroke/TIA associated with TAVR-specific procedural characteristics. Thus, a novel aspect of this study is the assessment of total number of balloon dilations and $\mathrm{CPB}$ as potential risk factors for TAVR-related cerebrovascular events. Furthermore, we developed a propensity score to help reduce any potential confounding bias.

PD emerged as one of the independent predictors of 30-day stroke/TIA after balloon-expandable TAVR, after adjusting for the propensity score, total number of balloon dilations, PVR and emergent CPB. The percentage of patients undergoing $\mathrm{PD}$ in the present study was similar to other studies. ${ }^{11} \mathrm{PD}$ is performed to reduce the degree of PVR, the latter of which is associated with increased longterm mortality. The findings of our study would suggest a trade-off between PVR and increased risk of cerebrovascular events in these patients. The incidence of stroke/ TIA in patients undergoing PD was $9.4 \%$ compared with $2.2 \%$ in patients without $\mathrm{PD}$, which translated into a fourfold relative increase in the risk of stroke/TIA. Similar findings were noted in another single-centre study, which reported an $11.9 \%$ incidence of 30-day stroke/TIA after PD compared with $2 \%$ in non-PD patients. However, the frequency of PD was twofold greater (28\%) in that study, as compared with our study $(14 \%) .{ }^{12}$ Another multicentre study of 1061 patients reported a 2.5-fold increased risk of stroke with PD after adjusting for other clinical risk factors. ${ }^{10}$ A post hoc analysis of the Placement of AoRtic TraNscathetER Valves (PARTNER) trial showed that PD was associated with an increased incidence of stroke only in the first 7 days, and no association was found with 30-day stroke. Although the 30-day incidence of stroke in PD patients was almost similar to our study $(4.9 \%$ vs $5.6 \%)$, the incidence of stroke in non-PD patients was higher in the PARTNER trial, which may explain the discrepant findings between these two studies $(3.4 \%$ vs $1.6 \%)$, respectively. ${ }^{11}$

Of note, the total number of balloon dilations during TAVR showed a trend towards increased risk of cerebrovascular events. However, they had no association after adjusting for PD, suggesting that $\mathrm{PD}$ may be the most important predictor of TAVR-related cerebrovascular events compared with prevalve and valve inflations. It is still unclear if the total number of postdilations had any impact on the incidence of clinical cerebrovascular events. In the current study, only 4 out of 53 patients underwent two or more postdilations and its effect could not be analysed because of the small number. Several factors may explain the findings. MRI-based studies have shown that $60 \%-70 \%$ of the patients undergoing TAVR have cerebral emboli after the procedure; however, only a few translate to clinical cerebrovascular events. ${ }^{67}$ A recent pathological study of the debris from embolic protection device during TAVR found that balloon-expandable valves were associated with tissue-derived debris in $79 \%$ of the patients. Importantly, PD showed a trend towards increased tissue embolisation compared with non-PD. ${ }^{20}$ We found that the CT calcium score was significantly higher in patients undergoing PD as compared with those who did not have PD, although it did not predict stroke/ TIA risk directly (see online supplemental tables 1 and 
2). It is conceivable that PD is associated with increased risk of clinical cerebrovascular events due to embolisation of calcium and tissue from the native aortic valve or the aortic wall. Additionally, RV pacing preballoon, during and postballoon-expandable valve deployment leads to a temporary tachycardic cardiac arrest resulting in cerebral hypoperfusion ${ }^{21}$ and possibly watershed infarcts in a minority of patients. It is also plausible that issues with patient-prosthesis mismatch, inappropriate valve sizing and repeated attempts ${ }^{22}$ at valve positioning and implantation may also increase the risk of stroke. ${ }^{17}$

One of the novel findings of this study was that emergent CPB during TAVR was associated with a significantly increased incidence of stroke/TIA at 30 days. In the bypass surgery literature, there is clear evidence that conversion of off-pump coronary artery bypass surgery to CPB was associated with increased risk of stroke. ${ }^{23}{ }^{24}$ Increased risk of stroke in these patients may be attributed to haemodynamic alterations and watershed infarcts, air/ fat embolism, scraping of atherosclerotic plaque and calcium or systemic inflammatory reaction. ${ }^{25}{ }^{26}$ Femoral CPB was used in 16 patients, out of which severe PVR was the reason in $25 \%$ cases. Retrograde perfusion with femoral CPB has been associated with an increased risk of stroke, ${ }^{27-29}$ although the data are not consistent among studies. ${ }^{30}$ The use of CPB was more frequent in patients who underwent $\mathrm{PD}$ as compared with those who did not undergo PD (see online supplemental table 2). Although both emergent CPB and PD were independent predictors of stroke/TIA in the final multivariable analysis, it is possible that a combination of these two factors played a role in some patients. Most of the cerebrovascular events in the emergent CPB group occurred in the first 4 days compared with those undergoing PD who continued to have stroke/TIA until 30 days. With improvement in technique and experience, the need for emergent rescue surgery and CPB after TAVR has declined. ${ }^{31}$ To further reduce the risk of stoke/TIA is the TAVR population, it is important to prevent complications that require CPB. In the present study, severe PVR postvalve deployment, transapical access and balloon valvuloplasty prior to valve deployment were the primary reasons for CPB. Therefore, meticulous preprocedural planning with optimal valve to annulus sizing, avoidance of pre-TAVR balloon valvuloplasty, early identification of complications and haemodynamic compromise may reduce the need for emergent CPB.

\section{Clinical implications}

The present study helps identify various procedural factors that are associated with the risk of cerebrovascular events in patients who underwent balloon-expandable TAVR. This also adds to the growing evidence that PD during TAVR is associated with a significantly increased risk of stroke/TIA in the first 30 days. The overall rate of 30-day stroke $(2.1 \%)$ and 30-day stroke/TIA (3.5\%) in the present study was lower than the randomised PARTNER clinical trials, ${ }^{18} 1932$ but similar to the national average reported recently. ${ }^{1}$ Moderate-to-severe PVR is associated with increased risk of mortality after TAVR, ${ }^{33}$ whereas data on association of mild PVR with mortality are mixed. ${ }^{33}$ PD may still be indicated in patients with more than moderate PVR to reduce long-term mortality; however, the increased risk of possible cerebrovascular events should be considered in those with mild PVR. Improvement in valve design with the SAPIEN 3 valve has reduced the incidence of PVR and need for PD during TAVR, which has translated into reduced incidence of 30-day stroke. ${ }^{35} \mathrm{CPB}$ offers an immediate rescue in patients with sudden haemodynamic instability, cardiogenic shock and those with low cardiac reserve. Larger studies should explore its association with stroke/TIA in TAVR patients.

\section{Limitations}

Our study has several limitations. This was a single-centre study from a tertiary care referral centre, and the data were analysed retrospectively. Therefore, inherent selection bias is possible. To reduce the bias associated with confounding, we performed a propensity score adjusted analysis. The total number of events was small precluding us from performing a temporal analysis or assessing the impact of operator experience with cerebrovascular events. Only clinically apparent events of stroke/TIA were evaluated by Neurology, which may have led to underestimation of cerebrovascular events. Data on specific types of delivery catheters were not recorded, so their impact on the cerebrovascular events could not be analysed. Ultrasound data on the degree of carotid atherosclerosis were not available, which may impact the risk of stroke/ TIA. The physicians performing and reading the intraoperative TEE varied from case to case and this could have led to heterogeneity in the interpretation of PVR degree. However, all the physicians were level III and trained in interventional echocardiography. Self-expanding transcatheter valve was not included in the present study because of small number of cases performed. This study included the older generation of balloon-expandable valves that were associated with a higher risk of stroke as compared with the newer-generation SAPIEN 3 valve. ${ }^{36}$

\section{CONCLUSION}

Postdilation as compared with total number of balloon dilations, and emergent CPB were the most important procedural risk factors associated with clinical cerebrovascular events in the first 30 days after balloon-expandable TAVR. Reduction in balloon postdilation with appropriate valve sizing may reduce the risk of stroke/TIA after TAVR.

Acknowledgements The authors appreciate the help of Rebecca M Severson for her help with data retrieval from the cardiovascular surgery database.

Contributors KG, RL and JPS had full access to all of the data in the study and take responsibility for the integrity of the data and the accuracy of the data analysis. Study concept and design: KG, DRH. Acquisition, analysis or interpretation of data: KG, JPS, RL, RDB. Drafting of the manuscript: all authors. Critical revision 
of the manuscript for important intellectual content: all authors. Statistical analysis: JPS, RL. Obtained funding: DRH. Administrative, technical or material support: KG, WTN, DRH. Study supervision: KG, DRH.

Funding The study was funded by the Department of Cardiovascular Diseases, Mayo Clinic.

Competing interests None declared.

Patient consent Not required.

Ethics approval The study was approved by the Mayo Clinic Institutional Review board.

Provenance and peer review Not commissioned; externally peer reviewed.

Open access This is an open access article distributed in accordance with the Creative Commons Attribution Non Commercial (CC BY-NC 4.0) license, which permits others to distribute, remix, adapt, build upon this work non-commercially, and license their derivative works on different terms, provided the original work is properly cited, appropriate credit is given, any changes made indicated, and the use is non-commercial. See: http://creativecommons.org/licenses/by-nc/4.0/.

\section{REFERENCES}

1. Holmes DR, Brennan JM, Rumsfeld JS, et al. Clinical outcomes at 1 year following transcatheter aortic valve replacement. JAMA 2015;313:1019-28.

2. Leon MB, Smith CR, Mack MJ, et al. Transcatheter or surgical aortic-valve replacement in intermediate-risk patients. $N$ Engl J Med 2016;374:1609-20.

3. Généreux P, Head SJ, Van Mieghem NM, et al. Clinical outcomes after transcatheter aortic valve replacement using valve academic research consortium definitions: a weighted meta-analysis of 3,519 patients from 16 studies. J Am Coll Cardiol 2012;59:2317-26.

4. Miller DC, Blackstone EH, Mack MJ, et al. Transcatheter (TAVR) versus surgical (AVR) aortic valve replacement: occurrence, hazard, risk factors, and consequences of neurologic events in the PARTNER trial. J Thorac Cardiovasc Surg 2012;143:832-43.

5. Fanning JP, Walters DL, Platts DG, et al. Characterization of neurological injury in transcatheter aortic valve implantation: how clear is the picture? Circulation 2014;129:504-15.

6. Rodés-Cabau J, Dumont E, Boone RH, et al. Cerebral embolism following transcatheter aortic valve implantation: comparison of transfemoral and transapical approaches. J Am Coll Cardiol 2011;57:18-28.

7. Arnold M, Schulz-Heise S, Achenbach S, et al. Embolic cerebral insults after transapical aortic valve implantation detected by magnetic resonance imaging. JACC Cardiovasc Interv 2010;3:1126-32

8. Grube E, Naber C, Abizaid A, et al. Feasibility of transcatheter aortic valve implantation without balloon pre-dilation: a pilot study. JACC Cardiovasc Interv 2011;4:751-7.

9. Vahanian A, Himbert D. Transcatheter aortic valve implantation: could it be done without prior balloon valvuloplasty? JACC Cardiovasc Interv 2011;4:758-9.

10. Nombela-Franco L, Webb JG, de Jaegere PP, et al. Timing, predictive factors, and prognostic value of cerebrovascular events in a large cohort of patients undergoing transcatheter aortic valve implantation. Circulation 2012;126:3041-53.

11. Hahn RT, Pibarot $P$, Webb J, et al. Outcomes with post-dilation following transcatheter aortic valve replacement: the PARTNER I tria (placement of aortic transcatheter valve). JACC Cardiovasc Interv 2014;7:781-9.

12. Nombela-Franco L, Rodés-Cabau J, DeLarochellière R, et al. Predictive factors, efficacy, and safety of balloon post-dilation after transcatheter aortic valve implantation with a balloon-expandable valve. JACC Cardiovasc Interv 2012;5:499-512.

13. Barbanti M, Petronio AS, Capodanno D, et al. Impact of balloon post-dilation on clinical outcomes after transcatheter aortic valve replacement with the self-expanding CoreValve prosthesis. JACC Cardiovasc Interv 2014;7:1014-21.

14. Shih T, Paone G, Theurer PF, et al. The society of thoracic surgeons adult cardiac surgery database version 2.73: more is better. Ann Thorac Surg 2015;100:516-21.
15. Agatston AS, Janowitz WR, Hildner FJ, et al. Quantification of coronary artery calcium using ultrafast computed tomography. J Am Coll Cardiol 1990;15:827-32.

16. Kappetein AP, Head SJ, Généreux P, et al. Updated standardized endpoint definitions for transcatheter aortic valve implantation: the Valve Academic Research Consortium-2 consensus document. J Am Coll Cardiol 2012;60:1438-54.

17. Athappan G, Gajulapalli RD, Sengodan P, et al. Influence of transcatheter aortic valve replacement strategy and valve design on stroke after transcatheter aortic valve replacement: a metaanalysis and systematic review of literature. J Am Coll Cardiol 2014;63:2101-10.

18. Leon MB, Smith CR, Mack M, et al. Transcatheter aortic-valve implantation for aortic stenosis in patients who cannot undergo surgery. N Engl J Med 2010;363:1597-607.

19. Smith CR, Leon MB, Mack MJ, et al. Transcatheter versus surgical aortic-valve replacement in high-risk patients. $N$ Engl $\mathrm{J}$ Med 2011;364:2187-98

20. Van Mieghem NM, El Faquir N, Rahhab Z, et al. Incidence and predictors of debris embolizing to the brain during transcatheter aortic valve implantation. JACC Cardiovasc Interv 2015;8:718-24.

21. Kahlert P, Knipp SC, Schlamann M, et al. Silent and apparent cerebral ischemia after percutaneous transfemoral aortic valve implantation: a diffusion-weighted magnetic resonance imaging study. Circulation 2010;121:870-8.

22. Stortecky S, Windecker S, Pilgrim T, et al. Cerebrovascular accidents complicating transcatheter aortic valve implantation: frequency, timing and impact on outcomes. Eurolntervention 2012;8:62-70.

23. Chowdhury R, White D, Kilgo P, et al. Risk factors for conversion to cardiopulmonary bypass during off-pump coronary artery bypass surgery. Ann Thorac Surg 2012;93:1936-42. discussion 42.

24. Jin R, Hiratzka LF, Grunkemeier GL, et al. Aborted off-pump coronary artery bypass patients have much worse outcomes than on-pump or successful off-pump patients. Circulation 2005;112(9 Suppl):I332-7.

25. Chen JW, Lin CH, Hsu RB. Mechanisms of early and delayed stroke after systematic off-pump coronary artery bypass. J Formos Med Assoc 2015;114:988-94.

26. Blauth $\mathrm{Cl}$. Macroemboli and microemboli during cardiopulmonary bypass. Ann Thorac Surg 1995;59:1300-3.

27. Cheng DC, Martin J, Lal A, et al. Minimally invasive versus conventional open mitral valve surgery: a meta-analysis and systematic review. Innovations 2011;6:84-103.

28. Gammie JS, Zhao Y, Peterson ED, et al. J. Maxwell Chamberlain Memorial Paper for adult cardiac surgery. Less-invasive mitral valve operations: trends and outcomes from the Society of Thoracic Surgeons Adult Cardiac Surgery Database. Ann Thorac Surg 2010:90:1401-8. discussion 08-10.

29. Murzi M, Cerillo AG, Miceli A, et al. Antegrade and retrograde arterial perfusion strategy in minimally invasive mitral-valve surgery: a propensity score analysis on 1280 patients. Eur J Cardiothorac Surg 2013;43:e167-e172.

30. Algarni KD, Suri RM, Daly RC. Robotic-assisted mitral valve repair: surgical technique. Multimed Man Cardiothorac Surg 2014;2014:mmu022.

31. Eggebrecht $\mathrm{H}$, Vaquerizo $\mathrm{B}$, Moris $\mathrm{C}$, et al. Incidence and outcomes of emergent cardiac surgery during transfemoral transcatheter aortic valve implantation (TAVI): insights from the European Registry on Emergent Cardiac Surgery during TAVI (EuRECS-TAVI). Eur Heart J 2018;39:676-84.

32. Makkar RR, Fontana GP, Jilaihawi H, et al. Transcatheter aortic-valve replacement for inoperable severe aortic stenosis. N Engl J Med 2012;366:1696-704.

33. Athappan G, Patvardhan E, Tuzcu EM, et al. Incidence, predictors, and outcomes of aortic regurgitation after transcatheter aortic valve replacement: meta-analysis and systematic review of literature. J Am Coll Cardiol 2013;61:1585-95.

34. Gilard $M$, Eltchaninoff $\mathrm{H}$, lung $B$, et al. Registry of transcatheter aortic-valve implantation in high-risk patients. $N$ Engl J Med 2012;366:1705-15

35. Webb J, Gerosa G, Lefèvre T, et al. Multicenter evaluation of a nextgeneration balloon-expandable transcatheter aortic valve. J Am Coll Cardiol 2014;64:2235-43.

36. Thourani VH, Kodali S, Makkar RR, et al. Transcatheter aortic valve replacement versus surgical valve replacement in intermediate-risk patients: a propensity score analysis. Lancet 2016;387:2218-25. 